\title{
EQUALIBRIUM POINTS OF RANDOM GENERALIZED GAMES
}

\author{
E. TARAFDAR \\ Department of Mathematics \\ The University of Queensland, Brisbane, Australia 4072 \\ XIAN-ZHI YUAN* \\ Department of Mathematics, Statistics and Computing Science \\ Dalhousie University, Halifax, Nova Scotia, Canada B3H 3J5 \\ and \\ Department of Mathematics \\ The University of Queensland, Brisbane, Australia 4072
}

(Received July 13, 1993 and in revised form August 30, 1995)

\begin{abstract}
In this paper, the concepts of random maximal elements, random equilibria and random generalized games are described. Secondly by measurable selection theorem, some existence theorems of random maximal elements for $L_{C}$-majorized correspondences are obtained. Then we prove existence theorems of random equilibria for non-compact one-person random games. Finally, a random equilibrium existence theorem for non-compact random generalized games (resp., random abstract economics) in topological vector spaces and a random equilibrium existence theorem of non-compact random games in locally convex topological vector spaces in which the constraint mappings are lower semicontinuous with countable number of players (resp., agents) are given. Our results are stochastic versions of corresponding results in the recent literatures.
\end{abstract}

KEY WORDS AND PHRASES. Fan - Browder fixed point, random equilibria, random abstract economy, generalized game, maximal element, $L_{c}$-majorized, measurable space and Suslin family.

1991 AMS SUBJECTION CLASSIFICATION. 28A20, 28B20, 47H04, 47H10, 47N10, 90A14, 90D06, 90D13.

\section{INTRODUCTION}

In the recent years, the classical Arrow-Debreu result [1] on the existence of Walrasian equilibrium has been generalized in many directions. Mas-Colell [15] has first shown that the existence of an equilibrium can be established without assuming preferences to be total or transitive. Next by using a maximal existence theorem, Gale and Mas-Colell [9] gave a proof of the existence of a competitive equilibrium without ordered preferences. By using Kakutain's fixed point theorem, Shafer and Sonnenschein [19] proved the powerful result on the 'Arrow-Debreu Lemma' for abstract economy in which preferences may not be total or transitive but have an open graph. At the same time, Borglin and Keiding [3] proved a new existence theorem for a compact abstract economy with KF-majorized preference correspondences. Following their ideas, there have been a number of generalizations of the existence of equilibria for compact abstract economies. For example, Ding et al [7], Tan and Yuan [20], Tan and Yuan [22-23], Tarafdar and Yuan [25], Toussaint [26], Tulcea [27-28] proved some very general equilibrium existence theorems for generalized games (abstract economies) with correspondences defined on a compact (resp., non-compact) strategy (choice) set of players (agents). These theorems generalized most known equilibrium theorems on compact generalized games due to Borglin and

- The corresponding address: X. Yuan, Department of Mathematics, The University of Queensland, Brisbane 4072, Australia; and E-mail: xzy Caxiom.maths.uq.ox.au 
Keiding [3], Shafer and Sonnenschein [19] and Yannelis and Prabhakar [30]. Unfortunately, all these results mentioned above are deterministic in nature, i.e., the equilibrium does not involve any random variable from measurable spaces.

On the other hand, Debreu discussed the uncertainty of behavior of an economic activity in the Chapter 7 of his book [5]. Since then, a series of papers concerning the uncertainty of behavior of economic actions have been published. For example, Hildenbrand [11] considered economy in which the preferences are random correspondence. Bewley [2] studied the existence of equilibrium in abstract economy with a measure space of agents and with an infinite-dimensional strategy space. Kim et al [12] also proved the existence of equilibria in abstract economy with a measure space of agents and with an infinite-dimensional strategy space by random fixed point theorems. In particular, Tan and Yuan [21] and Yannelis and Rustichini [29] also gave some existence theorems of random equilibria for random generalized games or random qualitative games recently under the various conditions.

In this paper we shall consider the following problem. The strategy set $X_{i}$ of the $i$ th players (resp., agents) is a nonempty subset of topological vector spaces, but the constraint and preferences of players (resp., agents) in generalized games (resp., abstract economics) are involved in the variable of a measurable space $(\Omega, \Sigma)$. Therefore, the equilibrium of generalized games which involves in a measurable space $(\Omega, \Sigma)$ should be a function of a random variable in the measurable space $(\Omega, \Sigma)$. More precisely, in mathematical language it is formulated as following: Let $I$ be a (possibly infinite) set of agents and $(\Omega, \Sigma)$ a measurable space. For each $i \in I$, let its strategy (resp., choice) set $X_{i}$ be a non-empty subset of a topological vector space, $X=\Pi_{i \in I} X_{i}$ the product space of $\left\{X_{i}\right\}_{i \in I}$ and $P_{i}: \Omega \times X \rightarrow 2^{X_{i}}$ a (set-valued) correspondence. Following the notion of Gale and Mas-Colell [10] in deterministic case, we have the following definition.

DEFINITION 1.1. The collection $\Gamma=\left(\Omega, X_{i}, P_{i}\right)_{i \in I}$ is said to be a random qualitative game. A measurable single-valued mapping $\psi: \Omega \rightarrow X$ is said to be a random equilibrium of the random qualitative game $\Gamma$ if

$$
P_{i}(\omega, \psi(\omega))=\emptyset
$$

for all $i \in I$ and all $\omega \in \Omega$.

DEFINITION 1.2. A random generalized game (resp., random abstract economy) is the family of collection $\Gamma=\left(\Omega, X_{i} ; A_{i}, B_{i} ; P_{i}\right)_{i \in I}$ where $I$ is a (finite or infinite) set of players (resp., agents) such that for each $i \in I, X_{i}$ is a non-empty subset of a topological vector space and $A_{i}, B_{i}: \Omega \times X=\Pi_{j \in I} X_{j} \rightarrow 2^{X_{i}}$ are (set-valued) constraint correspondences and $P_{i}: \Omega \times X \rightarrow 2^{X_{i}}$ is a set-valued preference correspondence (which are interpreted as for each player (or agent) $i \in I$, the associated constraint and preference correspondences $A_{i}, B_{i}$ and $P_{i}$ have stochastic actions). If the set $I$ of players (resp., agents) is finite, i.e., $I=\{1, \cdots, n\}$, where $n \in \mathbb{N}$ is a fixed positive integer, then $\Gamma=\left(\Omega ; X_{i} ; A_{i}, B_{i} ; P_{i}\right)_{i \in I}$ is also called an $N$-person random game.

DEFINITION 1.3. An random equilibrium of a random generalized game $\Gamma$ is a measurable mapping $\Omega \rightarrow X$ such that for each $i \in I$,

$$
\pi_{i}(\psi(\omega)) \in \overline{B_{i}}(\omega, \psi(\omega)) \text { and } A_{i}(\omega, \psi(\omega)) \cap P_{i}(\omega, \psi(\omega))=\emptyset
$$

for all $\omega \in \Omega$, where $\pi_{i}$ is the projection of $X$ onto $X_{i}$.

We would like to remark that if $A_{i}, B_{i}$ and $P_{i}$ of random generalized game $\Gamma=\left(\Omega ; A_{i}, B_{i} ; P_{i}\right)_{i \in I}$ are independent of variable $\omega \in \Omega$, i.e., $A_{i}(\omega, \cdot)=A_{i}(\cdot), B_{i}(\omega, \cdot)=B_{i}(\cdot)$ and $P_{i}(\omega, \cdot)=P_{i}(\cdot)$, when $\overline{B_{i}}(\hat{x})=c l_{X_{i}} B_{i}(\hat{x})$ (which is the case when $B_{i}$ has a closed graph in $X \times X_{i}$; in particular, when $c l B_{i}$ is upper semicontinuous with closed values), our definition of an equilibrium point coincides with that of Ding et al [7] in deterministic case; and if in addition, $A_{i}=B_{i}$ for each $i \in I$, our definition of an equilibrium point coincides with the standard definition in the deterministic case; e.g., see Borglin and Keiding [3], Tulcea [27] and Yannelis and Prabhakar [30]. 
This paper is organized as follows. The concepts of random maximal elements, random equilibria and random generalized games are described. Next by a measurable selection theorem due to Leese [14], a existence theorem of random maximal elements for $L_{C}$-majorized correspondences is obtained. Then we prove existence a theorem of random equilibria for non-compact random one-person games. Finally, a random equilibrium existence theorem for non-compact random generalized games in topological vector spaces and a random equilibrium existence theorem of random generalized games (resp., random abstract economics) in locally convex topological vector spaces in which the constraint mappings are lower semicontinuous and with countable number of players (resp., agents) are given. Our results are the stochastic versions of corresponding results in the literatures.

We now turn to explain some notions. The set of all real numbers is denoted by $\mathbb{R}$. Let $A$ be a subset of a topological space $X$. We shall denote by $2^{A}$ the family of all subsets of $A$, int $X(A)$ the interior of $A$ in $X$ and $c_{X}(A)$ the closure of $A$ in $X$. A subset $S$ of $X$ is said to be compactly open in $X$ if $S$ is relatively compact in each non-empty compact subset $C$ of $X$. If $S$ is a subset of a vector space $E$, we shall denote by $\operatorname{co} C$ the convex hull of $C$ in $E$. If $A$ is a nonempty subset of a topological vector space $E$ and $S, T: A \rightarrow 2^{E}$ are correspondences, then $c o T$, $T \cap S: A \rightarrow 2^{E}$ are correspondences defined by $(\operatorname{coT})(x)=\operatorname{coT}(x)$ and $(T \cap S)(x)=T(x) \cap S(x)$ for each $x \in A$, respectively. If $X$ and $Y$ are topological spaces and $(\Omega, \Sigma)$ is a measurable space, and $T: \Omega \times X \rightarrow 2^{Y}$ is a correspondence, the Graph of $T$ denoted by GraphT, is the set $\{(\omega, x, y) \in \Omega \times X \times Y: y \in T(\omega, x)\}$ and the correspondence $\bar{T}: \Omega \times X \rightarrow 2^{Y}$ is defined by $\bar{T}(\omega, x)=\left\{y \in Y:(x, y) \in c_{X \times Y} \operatorname{Graph} T(\omega, \cdot)\right\}$. For each fixed $\omega \in \Omega$, the graph of the mapping $T(\omega, \cdot): X \rightarrow 2^{Y}$ is defined by $\operatorname{Graph}(T(\omega, \cdot)=\{(x, y) \in X \times Y: y \in T(\omega, x)\}$, and the mapping $c l T: \Omega \times X \rightarrow 2^{Y}$ is defined by $c l T(\omega, x)=c l_{Y}(T(\omega, x))$ for each $(\omega, x) \in \Omega \times X$. It is easy to see that $\operatorname{cl} T(\omega, x) \subset \bar{T}(\omega, x)$ for each $(\omega, x) \in \Omega \times X$.

DEFINITION 1.4. Let $X$ be a topological space, $Y$ a non-empty subset of a vector space $E, \theta: X \rightarrow E$ a mapping and $\phi: X \rightarrow 2^{Y}$ a correspondence. Then

(1) $\phi$ is said to be of class $L_{\theta, C}$ (e.g., see Tan and Yuan [20]) if (a): for each $x \in X$, $\operatorname{co\phi } \phi(x) \subset Y$ and $\theta(x) \notin \operatorname{co\phi }(x)$ and (b): there exists a correspondence $\psi: X \rightarrow 2^{Y}$ such that for each $x \in X, \psi(x) \subset \phi(x)$, and $\psi^{-1}(y)$ is compactly open in $X$ for each $y \in Y$ and $\{x \in X: \phi(x) \neq \emptyset\}=\{x \in X: \psi(x) \neq \emptyset\}$

(2) $\left(\phi_{x}, \psi_{x} ; N_{x}\right)$ is an $L_{\theta, C}$-majorant of $\phi$ at $x$ if $\phi_{x}, \psi_{x}: X \rightarrow 2^{Y}$ two mappings and $N_{x}$ is an open neighborhood of $x$ in $X$ such that (a): for each $z \in N_{z}, \phi(z) \subset \phi_{z}(z)$ and $\theta(z) \notin \operatorname{co\phi } \phi_{x}(z)$; (b): for each $z \in X, \psi_{x}(z) \subset \phi_{x}(z)$ and $\operatorname{co} \phi_{z}(z) \subset Y$; and (c): for each $y \in Y, \psi_{x}^{-1}(y)$ is compactly open in $X$;

(3) $\phi$ is said to be of $L_{\theta, C}$-majorized if for each $x \in X$ with $\phi(x) \neq \emptyset$, there exists an

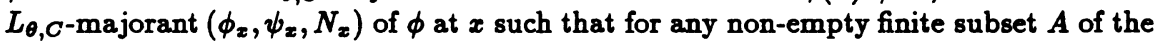
set $\{x \in X: \phi(x) \neq \emptyset\}$, we have the following equality:

$\left\{z \in \cap_{x \in A} N_{x}: \cap_{x \in A} \operatorname{co\phi } \phi_{z}(z) \neq \emptyset\right\}=\left\{z \in \cap_{x \in A} N_{x}: \cap_{x \in A} \cos \psi_{z}(z) \neq \emptyset\right\}$.

It is clear that every correspondence of class $L_{\theta, C}$ is $L_{\theta, C}$-majorized. We note that our notions of the correspondence $\phi$ being of class $L_{\theta, C}$ and $L_{\theta, C}$-majorized correspondence generalize the concepts of correspondence of classes $\mathcal{L}_{\theta}^{*}$ and $\mathcal{L}_{\theta}^{*}$-majorized introduced by Ding et al [7], which all in turn generalize the notions of $\phi \in C(X, Y, \theta)$ and $C$-majorized correspondence respectively introduced by Tulcea [27] and Yannelis and Prabhakar [30]. In this paper, we shall deal mainly with either the case (I) $X=Y$ and is a non-empty convex subset of the topological vector space $E$ and $\theta=I_{X}$, the identity mapping on $X$, or the case (II) $X=\Pi_{i \in I} X_{i}$ and $\theta=\pi_{j}: X \rightarrow X_{j}$ is the projection of $X$ onto $X_{j}$ and $Y=X_{j}$ is a non-empty convex subset of a topological vector space. In both cases (I) and (II), we shall write $L_{C}$ in place of $L_{\theta, C}$.

A measurable space $(\Omega, \Sigma)$ is a pair where $\Omega$ is a set and $\Sigma$ is a $\sigma$-algebra of subsets of $\Omega$. If $X$ is a set, $A \subset X$, and $\mathcal{D}$ is a non-empty family of subsets of $X$, we shall denote by $\mathcal{D} \cap A$ the family $\{D \cap A: D \in \mathcal{D}\}$ and by $\sigma_{X}(\mathcal{D})$ the smallest $\sigma$-algebra on $X$ generated by $\mathcal{D}$. If $X$ is a topological space with topology $\tau_{X}$, we shall use $\mathcal{B}(X)$ to denote $\sigma_{X}\left(\tau_{X}\right)$, the Borel $\sigma$-algebra on $X$ if there is no ambiguity on the topology $\tau_{X}$. If $(\Omega, \Sigma)$ and $(\Phi, \Gamma)$ are two measurable spaces, then $\Sigma \otimes \Gamma$ denotes the smallest $\sigma$-algebra on $\Omega \times \Phi$ which contains all the sets $A \times B$, where $A \in \Sigma, B \in \Gamma$, i.e. $\Sigma \otimes \Gamma=\sigma_{\Omega \times \Phi}(\Sigma \times \Gamma)$. We note that the Borel $\sigma$-algebra $\mathcal{B}\left(X_{1} \times X_{2}\right)$ 
contains $\mathcal{B}\left(X_{1}\right) \otimes \mathcal{B}\left(X_{2}\right)$ in general, but the converse is not necessarily true.

A function $f: \Omega \rightarrow \Phi$ is said to be $(\Sigma, \Gamma)$ measurable if $f^{-1}(B)=\{x \in \Omega: f(x) \in B\} \in \Sigma$ for each $B \in \Gamma$. Let $X$ be a topological space and $F:(\Omega, \Sigma) \rightarrow 2^{X}$ a correspondence. Then $F$ is said to be measurable (resp., weakly measurable) if $F^{-1}(B)=\{\omega \in \Omega: F(\omega) \cap B \neq \phi\} \in \Sigma$ for each closed (resp., open) subset $B$ of $X$. The mapping $F$ is said to have a measurable graph if Graph $F:=\{(\omega, y) \in \Omega \times X: y \in F(\omega)\} \in \Sigma \otimes \mathcal{B}(X)$. A function $f: \Omega \rightarrow X$ is said to be a measurable selection of $F$ if $f$ is a measurable function provided $f(\omega) \in F(\omega)$ for all $\omega \in \Omega$.

If $(\Omega, \Sigma),(\Phi, \Gamma)$ are measurable spaces and $Y$ is a topological space, then a mapping $F$ : $\Omega \times \Phi \rightarrow 2^{Y}$ is said to be jointly measurable (resp., jointly weakly measurable) if for every closed (resp., open) subset $B$ of $Y, f^{-1}(B) \in \Sigma \otimes \Gamma$. In the case $\Phi=X$ is a topological space, it is understood that $\Gamma$ is the Borel $\sigma$-algebra $\mathcal{B}(X)$. Let $\mu$ be a positive measure on a measurable space $(\Omega, \Sigma)$. A subset $N$ of $\Omega$ is called a $\mu$-negligible subset of $\Omega$ if there exists a measurable subset $A \in \Sigma$ such that $N \subset A$ and $\mu(A)=0$. The $\mu$-completion of $\Sigma$, denoted by $\Sigma_{\mu}$, is the $\sigma$-algebra on $\Omega$ generated by $\Sigma$ and the $\mu$-negligible subsets of $\Omega$. We know that each measure $\mu$ admits a unique extension $\hat{\mu}$ to $\Sigma_{\mu}$. The $\sigma$-algebra $\Sigma$ is said to be $\mu$-complete if $\Sigma=\Sigma_{\mu}$. Also, $(\Omega, \Sigma)$ is a complete measurable space if there is a positive measure $\mu$ on $(\Omega, \Sigma)$ such that $\boldsymbol{\Sigma}_{\mu}=\boldsymbol{\Sigma}$.

A topological space $X$ is said to be (i): a Polish space if $X$ is separable and metrizable by 'a complete metric; (ii) a Suslin space if $X$ is a Hausdorff topological space and the continuous image of a Polish space. A Polish (or Suslin) subset in a topological space is a subset which is a Polish (or Suslin) space. We note that the concept of 'Suslin set' plays very important roles in the study of measurable selection theory. We also remark that if $X_{1}$ and $X_{2}$ are Suslin spaces, then $\mathcal{B}\left(X_{1} \times X_{2}\right)=\mathcal{B}\left(X_{1}\right) \otimes \mathcal{B}\left(X_{2}\right)$; for more details, see Saint-Beuve [17, p.113].

DEFINITION 1.5. Denote by $\mathcal{J}$ and $\mathcal{F}$ the sets of infinite and finite sequences of positive integers respectively. Let $\mathcal{G}$ be a family of sets and $F: \mathcal{F} \rightarrow \mathcal{G}$ a mapping. For each $\sigma=$ $\left(\sigma_{i}\right)_{i=1}^{\infty} \in \mathcal{J}$ and $n \in \mathbb{N}$, we shall denote $\left(\sigma_{1}, \cdots, \sigma_{n}\right)$ by $\sigma / n$. Then $\bigcup_{\sigma \in \mathcal{J}} \bigcap_{n=1}^{\infty} F(\sigma / n)$ is said to be obtained from $\mathcal{G}$ by the Suslin operation. Now if every set obtained from $\mathcal{G}$ in this way is also in $\mathcal{G}$, then $\mathcal{G}$ is called a Suslin family.

Note that if $\mu$ is an outer measure on a measurable space $(\Omega, \Sigma)$, then $\Sigma$ is a Suslin family (e.g., see Saks [18, p.50]). In particular, if $(\Omega, \Sigma)$ is a complete measurable space, then $\Sigma$ is a Suslin family (for more details, see Wagner [32, p.864]). It also implies that the $\sigma$-algebra $\Sigma$ of Lebesgue measurable subsets of $[0,1]$ is a Suslin family.

DEFINITION 1.6. Let $X$ and $Y$ be topological spaces, $(\Omega, \Sigma)$ a measurable space and $F: \Omega \times X \rightarrow 2^{Y}$ a set-valued mapping. Then $F$ is said to be

(i) a random operator if for each fixed $x \in X$, the mapping $F(\cdot, x): \Omega \rightarrow 2^{Y}$ is a measurable mapping;

(ii) lower semicontinuous (resp., upper semicontinuous, continuous) if for each fixed $\omega \in \Omega$, $F(\omega, \cdot): X \rightarrow 2^{Y}$ is lower semicontinuous (resp., upper semicontinuous, continuous);

(iii) random upper semicontinuous (resp., random lower semicontinuous, random continuous) if $F$ is both a random operator and upper semicontinuous (resp., lower semicontinuous, continuous) mapping.

Let $A \subset X \times Y$. Throughout of this paper, we shall denote the projection of $A$ into $X$ by $\operatorname{Proj}_{X} A$ which is the set $\{x \in X$ : there exists some $y \in Y$ such that $(x, y) \in A\}$; and if $T: \Omega \times X \rightarrow 2^{Y}$, the domain of $T$, denoted by $\operatorname{Dom} T$, is the set $\{(\omega, x) \in \Omega \times X: T(\omega, x) \neq \emptyset\}$. It is easy to see that $\operatorname{Dom} T=\operatorname{Proj}_{\Omega \times X}$ GraphT. For the simplicity of our study in this paper, all topological spaces are assumed to be Hausdorff unless otherwise specified.

In the study of this paper, we shall need the following measurable selection theorem which is a Corollary of Theorem 7 of Leese in [14]:

THEOREM A. Let $(\Omega, \Sigma)$ be a measurable space, $\Sigma$ a Suslin family, $X$ a Suslin space and $F:(\Omega, \Sigma) \rightarrow 2^{X}$ be a mapping such that Graph $F \in \Sigma \otimes \mathcal{B}(X)$. Then there exists a sequence 
$\left\{\gamma_{n}\right\}_{n=1}^{\infty}$ of measurable selections of $F$ such that for each $\omega \in \Omega$, the set $\left\{\gamma_{n}(\omega), n=1,2, \cdots\right\}$ is dense in $F(\omega)$.

\section{RANDOM MAXIMAL ELEMENTS}

Before we prove existence theorems of random maximal elements, we shall need a non-compact version of the well-known Fan - Browder type fixed point theorem which has been recently established by Tan and Yuan [20] as applications of the classical Knaster - Kuratowski - Mazurkiewicz lemma (in short, KKM lemma which was first given in [13]). For the convenience of our study in this paper, we state it as

LEMMA 2.1. Let $X$ be a non-empty convex subset of a topological vector space $E$ (not necessarily Hausdorff) and $P, Q: X \rightarrow 2^{X}$ be such that

(a) for each $x \in X, P(x) \subset Q(x)$;

(b) for each $x \in X, P^{-1}(x)$ is compactly open in $X$;

(c) there exist a non-empty closed and compact subset $K$ of $X$ and $x_{0} \in X$ such that $X \backslash K \subset(\operatorname{coQ} Q)^{-1}\left(x_{0}\right)$

(d) for each $y \in K, P(y) \neq \emptyset$.

Then there exists an $x \in X$ such that $x \in \operatorname{coQ}(x)$.

PROOF. It is a special case of Theorem 2.4" of Tan and Yuan [20].

REMARK 2.1. We should like to remark that many generalizations of Fan-Browder type fixed point theorem have been given by many authors in recent years. For example, by posing another different non-compact condition on the mapping $P$ instead of mapping $\operatorname{co} Q$ in Lemma 2.1, the following non-compact version of Fan-Browder fixed point theorem has been established recently by Park [16, p.500]. In order to make a comparison with Lemma 2.1 above, we state here one of another non-compact version of Fan-Browder fixed point theorem which is essentially due to Park [16].

LEMMA 2.1'. Let $X$ be a non-empty convex subset of a topological vector space $E$ and $P, Q: X \rightarrow 2^{X}$ be such that

(a) for each $x \in X, P(x) \subset Q(x)$;

(b) for each $x \in X, P^{-1}(x)$ is compactly open in $X$;

(c) there exist a non-empty compact subset $K$ of $X$ such that for each non-empty finite subset $N$ of $X$, there exists a non-empty compact and convex subset $L_{N}$ of $X$ containing $N$ such that $L_{N} \backslash K \subset(c o P)^{-1}\left(L_{N}\right)$;

(d) for each $y \in K, P(y) \neq \emptyset$.

Then there exists an $x \in X$ such that $x \in \operatorname{co} Q(x)$.

PROOF. It is a special case of Theorem 5 of Park [16, p.500].

It is clear that Lemma 2.1 and Lemma 2.1 ' are independent to each other as the 'noncompact conditions (d)' in both Lemmas 2.1 and $2.1^{\prime}$ are different. Secondly, for more other recent results on the study of Fan-Browder type fixed point theorems and their applications to nonlinear analysis, convex analysis, mathematical economics, game theory and related topics, the interested readers can find more details and references from Park [16] and Yuan [31].

We also need the following selection result which is Lemma 3.1 of Tan and Yuan [20].

LEMMA 2.2. Let $X$ be a regular topological vector space and $Y$ a non-empty subset of a vector space $E$. Let $\theta: X \rightarrow E$ and $P: X \rightarrow 2^{Y}$ be $L_{\theta, C}$-majorized. If each open subset of $X$ containing the set $B=\{x \in X: P(x) \neq \emptyset\}$ is paracompact, then there exists a correspondence $\phi: X \rightarrow 2^{Y}$ of class $L_{\theta, C}$ such that $P(x) \subset \phi(x)$ for each $x \in X$.

PROOF. It is Lemma 3.1 of Tan and Yuan [20].

Now we have the following theorems concerning the existence of maximal elements.

THEOREM 2.3. Let $X$ be a non-empty convex subset of a topological vector space $E$ and 
$Q: X \rightarrow 2^{X}$ be of class $L_{I_{X}, C}$. Suppose that there exist a non-empty closed and compact subset $K$ of $X$ and $x_{0} \in X$ such that $x_{0} \in \operatorname{coQ}(y)$ for all $y \in X \backslash K$.

Then there exists a point $x \in K$ such that $Q(x)=0$.

PROOF. If the conclusion were false, then for each $x \in K, Q(x) \neq \emptyset$. Since $Q$ is of class $L_{I_{X}, C}$, let $P: X \rightarrow 2^{X}$ be a correspondence such that (a) for each $x \in X, P(x) \subset Q(x)$, (b) for each $y \in X, p^{-1}(y)$ is compactly open in $X$ and (c) $\{x \in X: p(x) \neq \emptyset\}=\{x \in X: Q(x) \neq \emptyset\}$. By Lemma 2.1, there exists a point $x \in X$ such that $x \in \operatorname{co} Q(x)$ which contradicts that $Q$ is of class $L_{I_{X}, C}$. Therefore the conclusion must hold.

THEOREM 2.4. Let $X$ be a non-empty paracompact convex subset of a topological vector

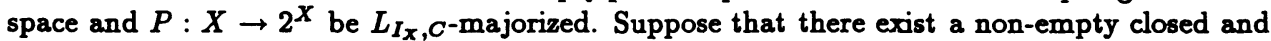
compact subset $K$ of $X$ and $x_{0} \in X$ such that $x_{0} \in \operatorname{coP}(y)$ for all $y \in X \backslash K$.

Then there exists a point $x \in K$ such that $P(x)=0$.

PROOF. Suppose that the conclusion does not hold. Then $P(x) \neq \emptyset$ for all $x \in X$ and hence the set $\{x \in X: P(x) \neq \emptyset\}=X$, which is paracompact. By Lemma 2.2, there exists a correspondence $\phi: X \rightarrow 2^{X}$ of class $L_{I_{X}, C}$ such that for each $x \in X, P(x) \subset \phi(x)$. Note that there exist a non-empty closed and compact subset $K$ of $X$ and $x_{0} \in X$ such that $x_{0} \in \operatorname{coP}(y) \subset$ $\operatorname{co\phi }(y)$ for all $y \in X \backslash K$. By Theorem 2.3, there exists a point $x \in K$ such that $\phi(x)=\emptyset$ so that $P(x)=\emptyset$ which is a contradiction. Therefore there must exist a point $x \in K$ such that $P(x)=\emptyset$, and we complete the proof.

Theorem 2.3 generalizes Corollary 1 of Borglin and Keiding [3], Theorem 2.2 of Toussaint [26], Theorem 2 of Tulcea [27] and Corollary 5.1 of Yannelis and Prabhakar [30].

Now we have the following existence theorems of random maximal elements which correspond to Theorem 2.3 and Theorem 2.4:

THEOREM 2.5. Let $(\Omega, \Sigma)$ be a measurable space with $\Sigma$ a Suslin family and $X$ be a non-empty convex Polish subset of a topological vector space and $Q: \Omega \times X \rightarrow 2^{X}$ be of class $L_{I_{X}, C}$ for each fixed $\omega \in \Omega$. Suppose that for any given $\omega \in \Omega$, there exist a non-empty closed and compact subset $K(\omega)$ of $X$ and $x_{0}(\omega) \in X$ such that $x_{0}(\omega) \in \operatorname{co} Q(\omega, y)$ for all $y \in X \backslash K(\omega)$, and $D o m Q \in \Sigma \otimes \mathcal{B}(X)$.

Then there exists a sequence $\left\{\psi_{n}\right\}_{n=1}^{\infty}$ of measurable mappings from $\Omega$ to $X$ such that for each $n=1,2, \cdots, Q\left(\omega, \psi_{n}(\omega)\right)=\emptyset$ for all $\omega \in \Omega$.

PROOF. Define $\Psi: \Omega \rightarrow 2^{X \times X}$ by $\Psi(\omega)=\{(x, x) \in X \times X: Q(\omega, x)=\emptyset\}$ for each $\omega \in \Omega$. Note that $Q(\omega, \cdot)$ satisfies all conditions of Theorem 2.3 for fixed $\omega \in \Omega$. Thus $\Psi(\omega) \neq \emptyset$ for each $\omega \in \Omega$. Let $\Delta:=\{(x, x) \in X \times X\}$. Then $\Delta \in \mathcal{B}(X \times X)$ as $\Delta$ is closed in $X \times X$, and we have $\Omega \times \Delta \in \Sigma \otimes \mathcal{B}(X \times X)$. Note that $\operatorname{Graph} \Psi=([(\Omega \times X) \backslash \operatorname{Dom} Q] \times X) \cap(\Omega \times \Delta)$ and $\operatorname{Dom} Q \in \Sigma \otimes \mathcal{B}(X)$, it follows that $G r a p h \Psi \in(\Sigma \otimes \mathcal{B}(X)) \otimes \mathcal{B}(X)=\Sigma \otimes \mathcal{B}(X \times X)$ by the fact that $X$ is a Suslin subset. Therefore $\Psi$ satisfies all conditions of Theorem $A$. By Theorem A, there exists a sequence $\left\{\psi_{n}^{\prime}\right\}_{n=1}^{\infty}$ of measurable selections of $\Psi$, where $\psi_{n}^{\prime}: \Omega \rightarrow X \times X$. But then for each $n=1,2, \cdots$, there exists $\psi_{n}: \Omega \rightarrow X$ such that $\psi_{n}^{\prime}(\omega)=\left(\psi_{n}(\omega), \psi_{n}(\omega)\right)$ for all $\omega \in \Omega$. Now if $A$ is a closed subset of $X$, then $A \times A$ is a closed subset of $X \times X$. Thus for each $n=1,2, \cdots$,

$$
\psi_{n}^{-1}(A)=\left\{\omega \in \Omega: \psi_{n}(\omega) \in A\right\}=\left\{\omega \in \Omega: \psi_{n}^{\prime}(\omega) \in(A \times A)\right\} \in \Sigma
$$

and hence each $\psi_{n}$ is measurable. Moreover, for each $i=1,2, \cdots, Q\left(\omega, \psi_{n}(\omega)\right)=\emptyset$ for all $\omega \in \Omega$.

By following the similar argument used in the proof of Theorem 2.5 and applying Theorem 2.4 instead of Theorem 2.3, we have the following existence theorem of random maximal elements.

THEOREM 2.6. Let $(\Omega, \Sigma)$ be a measurable space with $\Sigma$ a Suslin family and $X$ be a non-empty convex Polish subset of a topological vector space and $Q: \Omega \times X \rightarrow 2^{X}$ be such that for each fixed $\omega \in \Omega, Q(\omega, \cdot)$ is $L_{I_{X}, C}$-majorized for each fixed $\omega \in \Omega$. Suppose that for any given $\omega \in \Omega$, there exist a non-empty closed and compact subset $K(\omega)$ of $X$ and $x_{0}(\omega) \in X$ 
such that $x_{0}(\omega) \in \operatorname{co} Q(\omega, y)$ for all $y \in X \backslash K(\omega)$ and $D o m Q \in \Sigma \otimes \mathcal{B}(X)$. Then there exists a sequence $\left\{\psi_{n}\right\}_{n=1}^{\infty}$ of measurable mappings from $\Omega$ to $X$ such that such that for each $i=1,2, \cdots$, $Q\left(\omega, \psi_{n}(\omega)\right)=\emptyset$ for all $\omega \in \Omega$.

\section{RANDOM EQUILIBRIA IN TOPOLOGICAL VECTOR SPACES}

As an application of Theorem A, we shall first prove the following existence theorem of a random equilibrium for a one-person random game. Then general existence theorems of random equilibria for random generalized games (resp., random abstract economies) will be established.

THEOREM 3.1. Let $(\Omega, \Sigma)$ be a measurable space with $\Sigma$ a Suslin family and $X$ a nonempty convex Polish subset of a topological vector space $E$. Suppose $A, B, P: \Omega \times X \rightarrow 2^{X}$ are such that

(i) for each $(\omega, x) \in \Omega \times X, A(\omega, x)$ is non-empty and $c o(A(\omega, x)) \subset B(\omega, x)$;

(ii) for any given $\omega \in \Omega$, for each $y \in X, A_{\omega}^{-1}(y)=\{x \in X: y \in A(\omega, x)\}$ is compactly open in X;

(iii) for each fixed $\omega \in \Omega, A(\omega, \cdot) \cap P(\omega, \cdot)$ is of class $L_{C}$;

(iv) $\operatorname{Dom}(A \cap P) \in \Sigma \otimes \mathcal{B}(X)$ and $G \operatorname{raph} \bar{B} \in \Sigma \otimes \mathcal{B}(X \times X)$;

(v) for any given $\omega \in \Omega$, there exist a non-empty closed and compact subset $K(\omega)$ of $X$ and $x_{0}(\omega) \in X$ such that $x_{0}(\omega) \in c o(A(\omega, y) \cap P(\omega, y))$ for all $y \in X \backslash K(\omega)$.

Then there exists a sequence $\left\{\psi_{n}\right\}_{n=1}^{\infty}$ of measurable mappings from $\Omega$ to $X$ such that for each $n=1,2, \cdots,\left(\psi_{n}(\omega) \in \bar{B}\left(\omega, \psi_{n}(\omega)\right)\right.$ and $A\left(\omega, \psi_{n}(\omega)\right) \cap P\left(\omega, \psi_{n}(\omega)\right)=\emptyset$ for all $\omega \in \Omega$.

PROOF. Define $\Psi: \Omega \rightarrow 2^{X \times X}$ by

$$
\Psi(\omega)=\{(x, x) \in X \times X: A(\omega, x) \cap P(\omega, x)=\emptyset \text { and } x \in \bar{B}(\omega, x)\}
$$

for each $\omega \in \Omega \times X$. Then Theorem 4.1 of Tan and Yuan [20] implies that for each $\omega \in \Omega$, there exists $x_{\omega} \in X$ such that $\left(x_{\omega}, x_{\omega}\right) \in \Psi(\omega)$. Note that

$$
\operatorname{Graph} \Psi=\{[(\Omega \times X) \backslash \operatorname{Dom}(A \cap P)] \times X\} \cap\{\operatorname{Graph} \bar{B} \cap(\Omega \times \Delta)\},
$$

where $\Delta=\{(x, x) \in X \times X: x \in X\}$. By our hypotheses, $\operatorname{Dom}(A \cap P) \in \Sigma \otimes \mathcal{B}(X)$ and Graph $\bar{B} \in \Sigma \otimes \mathcal{B}(X \times X)$, it follows that

$$
\operatorname{Graph} \Psi \in[\Sigma \otimes \mathcal{B}(X) \times \mathcal{B}(X)] \cap[\Sigma \otimes \mathcal{B}(X \times X)]=\Sigma \otimes \mathcal{B}(X \times X)
$$

as $X$ is a Suslin set. Therefore $\Psi$ satisfies all conditions of Theorem A. By Theorem A, there exists a sequence $\left\{\psi_{n}^{\prime}\right\}_{n=1}^{\infty}$ of measurable selections of $\Psi$, where $\psi_{n}^{\prime}: \Omega \rightarrow X \times X$. But then for each $n=1,2, \cdots$, there exists $\psi_{n}: \Omega \rightarrow X$ such that $\psi_{n}^{\prime}(\omega)=\left(\psi_{n}(\omega), \psi_{n}(\omega)\right)$ for all $\omega \in \Omega$. Now by following the same approach used in the proof in the Theorem 2.5, we have that each $\psi_{n}$ is measurable; and moreover, for each $i=1,2, \cdots, A\left(\omega, \psi_{n}(\omega)\right) \cap P\left(\omega, \psi_{n}(\omega)\right)=\emptyset$ and $\psi_{n}(\omega) \in \bar{B}\left(\psi_{n}(\omega)\right)$ for all $\omega \in \Omega$.

Before we deduce the random equilibrium existence theorem for a non-compact random generalized game (resp., random abstract economy) with a countable number of players (resp., agents). We recall the following result.

LEMMA 3.2. Let $\Gamma=\left(X_{i} ; A_{i}, B_{i} ; P_{i}\right)_{i \in I}$ be a generalized game such that $X=\Pi_{i \in I} X_{i}$ is paracompact. Suppose that the following conditions are satisfied for each $i \in I$ :

(i) $X_{i}$ is a non-empty convex subset of a topological vector space $F_{i}$;

(ii) for each $x \in X, A_{i}(x)$ is non-empty, $\operatorname{co} A_{i}(x) \subset B_{i}(x)$;

(iii) for each $y \in X_{i}, A_{i}^{-1}(y)$ is compactly open in $X$;

(iv) $A_{i} \cap P_{i}$ is $L_{C}$-majorized;

(v) the set $E_{i}=\left\{x \in X: A_{i}(x) \cap P_{i}(x) \neq \emptyset\right\}$ is open in $X$;

(vi) there exist a non-empty closed and compact subset $K$ of $X$ and $x^{0}=\left(x_{i}^{0}\right)_{i \in I} \in X$ such that $x_{i}^{0} \in \operatorname{co}\left(A_{i}(y) \cap P_{i}(y)\right)$ for all $y \in X \backslash K$. 
Then $\Gamma$ has an equilibrium in $K$.

PROOF. This is a special case of Theorem 4.3 of Tarafdar and Yuan [25] (and see also Theorem 5.3 of Ding and Tarafdar [8]).

Now we have the following:

THEOREM 3.3. Let $(\Omega, \Sigma)$ be a measurable space with $\Sigma$ a Suslin family and $\Gamma=$ $\left(\Omega ; X_{i} ; A_{i}, B_{i} ; P_{i}\right)_{i \in I}$ be a random generalized game such that $I$ is countable and the following conditions are satisfied for each $i \in I$ :

(i) $X_{i}$ is a non-empty convex Polish subset of a topological vector space $F_{i}$;

(ii) for each $(\omega, x) \in \Omega \times X, A_{i}(\omega, x)$ is non-empty, $\operatorname{co} A_{i}(\omega, x) \subset B_{i}(\omega, x)$;

(iii) for any given $\omega \in \Omega$, for each $y \in X_{i}, A_{\omega, i}^{-1}(y)=\left\{x \in X: y \in A_{i}(\omega, x)\right\}$ is compactly open in $X$

(iv) $A_{i}(\omega, \cdot) \cap P_{i}(\omega, \cdot)$ is $L_{C}$-majorized for each fixed $\omega \in \Omega$ and $\operatorname{Dom}\left(A_{i} \cap P_{i}\right)$, and $G r a p h \bar{B} \epsilon$ $\Sigma \otimes \mathcal{B}(X \times X)$, where the mapping $B: \Omega \times X \rightarrow 2^{X}$ defined by $\bar{B}(\omega, x)=\Pi_{i \in I} \overline{B_{i}}(\omega, x)$ for each $(\omega, x) \in \Omega \times X$.

(v) the set $E_{i}(\omega)=\left\{x \in X: A_{i}(\omega, x) \cap P_{i}(\omega, x) \neq \emptyset\right\}$ is open in $X$ for each $\omega \in \Omega$;

(vi) for any given $\omega \in \Omega$, there exist a non-empty closed and compact subset $K(\omega)$ of $X$ and $x^{0}(\omega)=\left(x_{i}^{0}(\omega)\right)_{i \in I} \in X$ such that $x_{i}^{0}(\omega) \in \operatorname{co}\left(A_{i}(\omega, y) \cap P_{i}(\omega, y)\right)$ for all $y \in X \backslash K(\omega)$.

Then $\Gamma$ has a sequence $\left\{\psi_{n}\right\}_{n=1}^{\infty}$ of measurable mappings from $\Omega$ to $X$ such that for each $n=1,2, \cdots, \pi_{i}\left(\psi_{n}(\omega)\right) \in \overline{B_{i}}\left(\omega, \psi_{n}(\omega)\right)$ and $A_{i}\left(\omega, \psi_{n}(\omega)\right) \cap P_{i}\left(\omega, \psi_{n}(\omega)\right)=\emptyset$ for all $\omega \in \Omega$ and all $i \in I$.

PROOF. For each $i \in I$, define $\Psi_{i}: \Omega \rightarrow 2^{X \times X}$ by $\Psi_{i}(\omega)=\left\{(x, x) \in X \times X: A_{i}(\omega, x) \cap\right.$ $P_{i}(\omega, x)=\emptyset$ and $\left.x_{i} \in \overline{B_{i}}(\omega, x)\right\}$ and $\Psi: \Omega \rightarrow 2^{X \times X}$ by $\Psi(\omega)=\Pi \Psi_{i=1}^{\infty}(\omega)$ for each $\omega \in \Omega$. Then $\Psi(\omega) \neq \emptyset$ for each fixed $\omega \in \Omega$ by our hypotheses of (i)-(v) and Lemma 3.2. Let $\Delta=$ $\{(x, x) \in X \times X: x \in X\}$. Then $G r a p h \Psi=\cap_{i \in I} G r a p h \Psi=\left[\left(\Omega \times X \backslash\left[\cup_{i \in I} \operatorname{Dom}\left(A_{i} \cap P_{i}\right)\right]\right) \times\right.$ $X] \cap[G r a p h \bar{B} \cap(\Omega \times \Delta)]$. Note that $\operatorname{Graph} \bar{B} \in \Sigma \otimes \mathcal{B}(X \times X)$ and $\operatorname{Dom}\left(A_{i} \cap P_{i}\right) \in \Sigma \otimes \mathcal{B}(X)$ for each $i \in I$, so that $\cup_{i \in I}\left(\operatorname{Dom}\left(A_{i} \cap P_{i}\right)\right) \in \Sigma \otimes \mathcal{B}(X)$ since $I$ is countable. Therefore Graph $\Psi \in \Sigma \otimes \mathcal{B}(X \times X)$. Hence $\Psi$ satisfies all conditions of Theorem $\mathrm{A}$. By Theorem $\mathrm{A}$, there exists a sequence $\left\{\psi_{n}^{\prime}\right\}_{n=1}^{\infty}$ of measurable selections of $\Psi$, where $\psi_{n}^{\prime}: \Omega \rightarrow X \times X$. But then for each $n=1,2, \cdots$, there exists $\psi_{n}: \Omega \rightarrow X$ such that $\psi_{n}^{\prime}(\omega)=\left(\psi_{n}(\omega), \psi_{n}(\omega)\right)$ for all $\omega \in \Omega$. Now by the same proof in Theorem 2.5, it is clear that each $\psi_{n}$ is measurable. Moreover, for each $n=1,2, \cdots, A_{i}\left(\omega, \psi_{n}(\omega)\right) \cap P_{i}\left(\omega, \psi_{n}(\omega)\right)=\emptyset$ and $\pi_{i}\left(\psi_{n}(\omega)\right) \in \overline{B_{i}}\left(\omega, \psi_{n}(\omega)\right)$ for all $\omega \in \Omega$ and $i \in I$.

REMARK 3.1. In Theorem 3.3, if $G r a p h \overline{B_{i}} \in \Sigma \otimes \mathcal{B}\left(X \times X_{i}\right)$ for each $i \in I$, then the mapping $\bar{B}: \Omega \times X \rightarrow 2^{X}$ defined by $\bar{B}(\omega, x)=\Pi_{i \in I} \overline{B_{i}}(\omega, x)$ for each $i \in I$ has a measurable graph, i.e., $G r a p h \bar{B} \in \Sigma \otimes \mathcal{B}\left(X \times X_{i}\right)$. We would also like to address that Theorem 3.3 is a stochastic version of Theorem 4.3 of Tarafdar and Yuan [25] which, in turn includes Theorem 5.3 of Ding and Tarafdar [8] as a special case when the set $I$ of players is countable.

As an immediate consequence of Theorem 3.3, we have the following existence result.

COROLLARY 3.4. Let $(\Omega, \Sigma)$ be a measurable space with $\Sigma$ a Suslin family. $\Gamma=$ $\left(\Omega ; X_{i} ; A_{i}, B_{i} ; P_{i}\right)_{i \in I}$ be a random generalized game such that $X=\Pi_{i \in I} X_{i}$ is paracompact. Suppose that $I$ is countable and the following conditions are satisfied for each $i \in I$ :

(i) $X_{i}$ is a non-empty convex Polish subset of a topological vector space $F_{i}$;

(ii) for each $(\omega, x) \in \Omega \times X,\left(A_{i}(\omega, x)\right.$ is non-empty and $\operatorname{coA}_{i}(\omega, x) \subset B_{i}(\omega, x)$; and $\operatorname{Dom}\left(A_{i} \cap P_{i}\right)$ and $G r a p h \bar{B}_{i} \in \Sigma \otimes \mathcal{B}\left(X \times X_{i}\right)$

(iii) for any given $\omega \in \Omega$, each $y \in X_{i}, A_{\omega, i}^{-1}(y)=\left\{x \in X: y \in A_{i}(\omega, x)\right\}$ and $P_{\omega, i}^{-1}(y)=\{x \in$ $\left.X: y \in P_{i}(\omega, x)\right\}$ are open in $X$

(iv) for any given $\omega \in \Omega$, for each $x \in X, x_{i} \notin \operatorname{co} P_{i}(\omega, x)$;

(v) for any given $\omega \in \Omega$, there exist a non-empty closed and compact subset $K(\omega)$ of $X$ and $x^{0}(\omega)=\left(x_{i}^{0}(\omega)\right)_{i \in I} \in X$ such that $x_{i}^{0}(\omega) \in \operatorname{co}\left(A_{i}(\omega, y) \cap P_{i}(\omega, y)\right)$ for all $y \in X \backslash K(\omega)$. 
Then $\Gamma$ has a sequence of random equilibrium $\left\{\psi_{n}\right\}_{n=1}^{\infty}$ from $\Omega$ to $X$ such that for all $i \in I$, $\pi_{i}\left(\psi_{n}(\omega)\right) \in \overline{B_{i}}\left(\omega, \psi_{n}(\omega)\right)$ and $A_{i}\left(\omega, \psi_{n}(\omega)\right) \cap P_{i}\left(\omega, \psi_{n}(\omega)\right)=\emptyset$ for all $\omega \in \Omega$.

PROOF. Note that for each fixed $\omega \in \Omega$, the set $\left\{x \in X: A_{i}(\omega, x) \cap P_{i}(\omega, x) \neq \emptyset\right\}=$ $\cup_{y \in X_{i}}\left(A_{\omega, i}^{-1}(y) \cap P_{\omega, i}^{-1}(y)\right)$ which is open by (iii). Thus all hypotheses of Theorem 3.3 are satisfied. By Theorem 3.3, the conclusion follows.

Corollary 3.4 is also stochastic version of Corollary 4.4 of Tan an Yuan [20] which in turn generalizes Theorem 2.5 of Toussaint [26], Corollary 2 of Tulcea [27] (also Corollary 2 in [28]) and and Theorem 6.1 of Yannelis and Prabhakar [30] to non-compact generalized games with $I$ is countable.

\section{RANDOM EQUILIBRIA IN LOCALLY CONVEX SPACES}

Finally, by the same argument as employed in Theorem 3.3 and the existence theorem of equilibria of generalized games in locally convex topological spaces (i.e., Theorem 5.4 of Tarafdar and Yuan [25] (see also Theorem 5.6 of Ding and Tarafdar [8]), we have the following random equilibrium existence theorem for a random generalized game (resp., random abstract economy) with countable number of players (resp., agents) in locally convex topological vector space.

THEOREM 4.1. Let $(\Omega, \Sigma)$ be a measurable space with $\Sigma$ a Suslin family and $\Gamma=$ $\left(\Omega ; X_{i} ; A_{i}, B_{i} ; P_{i}\right)_{i \in I}$ a random generalized game such that $I$ is countable and the following conditions are satisfied for each $i \in I$ :

(i) $X_{i}$ is a non-empty convex Polish subset of a locally convex vector space $F_{i}$;

(ii) for each $\omega \in \Omega, A_{i}(\omega, \cdot): X \rightarrow 2^{X_{i}}$ is lower semicontinuous and for each $(\omega, x) \in \Omega \times X$, $A_{i}(\omega, x)$ is non-empty and $\operatorname{coA}_{i}(\omega, x) \subset B_{i}(\omega, x)$; and $\operatorname{Dom}\left(A_{i} \cap P_{i}\right)$ and $G r a p h \overline{B_{i}} \in$ $\Sigma \otimes \mathcal{B}\left(X \times X_{i}\right)$

(iii) for each $\omega \in \Omega, A_{i}(\omega, \cdot) \cap P_{i}(\omega, \cdot)$ is $L_{C}$-majorized;

(iv) for each given $\omega \in \Omega$, the set $E_{i}(\omega)=\left\{x \in X: A_{i}(\omega, x) \cap P_{i}(\omega, x) \neq \emptyset\right\}$ is open in $X$;

(v) for any given $\omega \in \Omega$, there exist a non-empty closed and compact subset $K(\omega)$ of $X$ and $x^{0}(\omega)=\left(x_{i}^{0}(\omega)\right)_{i \in I} \in X$ such that $x_{i}^{0}(\omega) \in \operatorname{co}\left(A_{i}(\omega, y) \cap P_{i}(\omega, y)\right)$ for all $y \in X \backslash K(\omega)$.

Then $\Gamma$ has a sequence $\left\{\psi_{n}\right\}_{n=1}^{\infty}$ of measurable mappings from $\Omega$ to $X$ such that for each $n=1,2, \cdots, \pi_{i}\left(\psi_{n}(\omega)\right) \in \overline{B_{i}}\left(\omega, \psi_{n}(\omega)\right)$ and $A_{i}\left(\omega, \psi_{n}(\omega)\right) \cap P_{i}\left(\omega, \psi_{n}(\omega)\right)=\emptyset$ for all $\omega \in \Omega$ and for all $i \in I$.

PROOF. For each $i \in I$, define $\Psi_{i}: \Omega \rightarrow 2^{X \times X}$ by $\Psi_{i}(\omega)=\left\{(x, x) \in X \times X: A_{i}(\omega, x) \cap\right.$ $P_{i}(\omega, x)=\emptyset$ and $\left.x_{i} \in \overline{B_{i}}(\omega, x)\right\}$ and $\Psi: \Omega \rightarrow 2^{X \times X}$ by $\Psi(\omega)=\cap \Psi_{i=1}^{\infty}(\omega)$ for each $\omega \in \Omega$. Then $\Psi(\omega) \neq \emptyset$ for each fixed $\omega \in \Omega$ by our assumptions (1)-(v) and Theorem 5.4 of Tarafdar and Yuan [25] (see also Theorem 5.6 of Ding and Tarafdar [8]). Let $\Delta=\{(x, x) \in X \times X: x \in X\}$. Define a mapping $B: \Omega \times X \rightarrow 2^{X}$ by $B(\omega, x)=\Pi_{i \in I} \bar{B}_{i}(\omega, x)$ for each $(\omega, x) \in \Omega \times X$. Since for each $i \in I, G r a p h \overline{B_{i}} \in \Sigma \otimes \mathcal{B}\left(X \times X_{i}\right)$, then it is easily to see that $G r a p h \bar{B} \in \Sigma \otimes \mathcal{B}(X \times X)$. Note that Graph $\Psi=\cap_{i \in I} \operatorname{Graph} \Psi=\left[\left(\Omega \times X \backslash\left[\cup_{i \in I} \operatorname{Dom}\left(A_{i} \cap P_{i}\right)\right]\right) \times X\right] \cap[\operatorname{Graph} \bar{B} \cap(\Omega \times \Delta)]$ and $\operatorname{Graph} \bar{B} \in \Sigma \otimes \mathcal{B}(X \times X)$, since $\operatorname{Dom}\left(A_{i} \cap P_{i}\right) \in \Sigma \otimes \mathcal{B}(X)$ for each $i \in I$ and $I$ is countable, $\cup_{i \in I}\left(\operatorname{Dom}\left(A_{i} \cap P_{i}\right)\right) \in \Sigma \otimes \mathcal{B}(X)$. Therefore Graph $\Psi \in \Sigma \otimes \mathcal{B}(X \times X)$. Hence $\Psi$ satisfies all conditions of Theorem $A$. By Theorem $A$, there exists a sequence $\left\{\psi_{n}^{\prime}\right\}_{n=1}^{\infty}$ of measurable selections of $\Psi$, where $\psi_{n}^{\prime}: \Omega \rightarrow X \times X$. But then for each $n=1,2, \cdots$, there exists $\psi_{n}: \Omega \rightarrow X$ such that $\psi_{n}^{\prime}(\omega)=\left(\psi_{n}(\omega), \psi_{n}(\omega)\right)$ for all $\omega \in \Omega$. Now following the same proof in Theorem 2.5, it is clear that each $\psi_{n}$ is measurable. Moreover, for each $n=1,2, \cdots$, $A_{i}\left(\omega, \psi_{n}(\omega)\right) \cap P_{i}\left(\omega, \psi_{n}(\omega)\right)=\emptyset$ and $\pi_{i}\left(\psi_{n}(\omega)\right) \in \overline{B_{i}}\left(\omega, \psi_{n}(\omega)\right)$ for all $\omega \in \Omega$ and for all $i \in I$.

Theorem 4.1 is also a stochastic version of Theorem 5.4 of Tarafdar and Yuan [25] (which in turn, generalizes Theorem 5.6 of Ding and Tarafdar [8], Corollary 3 of Borglin and Keiding [3, p.315], Theorem 4.1 of Chang [4, p.247] and Theorem of Shafer and Sonnenschein [19, p.374] with countable number of players (resp., agents). For other kinds of existence theorems of random equilibria for random generalized games, we refer the interested readers to Tan and Yuan [21] and Yannelis and Rustichini [29]. 
ACKNOWLEDGMENT. Both authors would like to thank referee for his useful suggestions which lead to the present version of this paper.

\section{REFERENCES}

1. ARROW, K. J. and DEBREU, G., Existence of an Equilibrium for a Competitive Economy, Econometrica 22 (1954), 265-290.

2. BEWLEY, T., Existence of Equilibrium with Infinitely Many Commodities, J. Econom. Theory 4 (1972), 514-540.

3. BORGLIN, A. and KEIDING, H., Existence of Equilibrium Actions of Equilibrium, 'A note the 'new' existence theorems', J. Math. Economics 3 (1976), 313-316.

4. CHANG, Y., On the Nash Equilibrium, Soochow J. Math. 16 (1990), 241-248.

5. DEBREU, G., Theory of Value: An Axiomatic Analysis of Economic Equilibria, New Haven and London, Yale University Press, 1959.

6. DEBREU, G., Integration of Correspondences, Proc. Fifth Berkeley Sympos. Math. Stat. \& Probability (L. Lecam, J. Neyman, and E. L. Scott, eds.), vol. II, Part I, Univ. Calif. Press, Berkeley, 1967, pp. 351-372.

7. DING, X. P., KIM, W. K. and TAN, K. K., Equilibria of Non-compact Generalized Games with $L^{*}$-Majorised Preference Correspondences, J. Math. Anal. Appl. (1992), 508-517.

8. DING, X. P. and TARAFDAR, E, Fixed Point Theorems and Existence of Equilibrium Points of Noncompact Abstract Economies, Nonlinear World 1, 319-340.

9. GALE, D. and MAS-COLELL, A, An Equilibrium Existence for a General Model Without Ordered Preferences, J. Math. Econom. 2 (1975), 9-15.

10. GALE, D and MAS-COLELL, A, On the Role of Complete, Translative Preferences in Equilibrium Theory, Equilibrium and Disequilibrium in Economics Theory (G. Schwödiauer, ed.), Reidel, Dordrecht, 1978, pp. 7-14.

11. HILDENBRAND, W., Random Preferences and Equilibrium Analysis, J. Econ. Theory 3 (1971), 414-429.

12. KIM, T., PRIKRY, K. and YANNELIS, N., Caratheodory-type Selections and Random Fixed Point Theorems, J. Math. Anal. Appl. 122 (1987), 393-407.

13. KNASTER, B., KURATOWSKI, C. and MAZURKIEWICZ, S., Ein Beweis des Fixpunktsatses für n dimensionale Simplexe, Fund. Math. 14 (1929), 132-137.

14. LEESE, J., Multifunctions of Souslin Type, Bull. Austral. Math. Soc. 11 (1974), 395-411.

15. MAS-COLELL, A., An Equilibrium Existence Theorem without Complete or Transitive Preferences, J. Math. Econom. 1 (1974), 237-246.

16. PARK, S., Foundations of KKM Theory via Coincidences of Composites of Upper Semicontinuous Maps, J. Korean Math. Soc. 81 (1994), 493-519.

17. SAINT-BEUVE, M. F., On the Existence of von Neumann-Aumann's Theorem, J. Punct. Anal. 17 (1974), 112-129.

18. SAKS, S., Theory of the Integral, Dover, New York, 1968.

19. SHAFER, W. and SONNENSCHEIN, H., Equilibrium in Abstract Economies without Ordered Preferences, J. Math. Econ. 2 (1975), 345-348.

20. TAN, K. K. and YUAN, X. Z., A Minimax Inequality with Application to Existence of Equilibrium Points, Bull. Austral. Math. Soc. 47 (1993).

21. TAN, K. K. and YUAN, X. Z., Equilibria of Random Generalised Games, Nonlinear World 1 (1994), 187-199.

22. TAN, K. K. and YUAN, X. Z., Equilibria of Generalised Games with U-Majorised Preference Correspondences, Economics Letters 41 (1993), 379-383.

23. TAN, K. K. and YUAN, X. Z., Existence of Equilibrium for Abstract Economies, J. Math. Economics 28 (1994), 243-251.

24. TARAFDAR, E., On Nonlinear Variational Inequalities, Proc. Amer. Math. Soc. 67 (1977), 95-98.

25. E. TARAFDAR and YUAN, X. Z., The Study of Equilibria for Abstract Economics in Topological Vector Spaces - A Unified Approach, Presented in The 7th World Congress of Econometrics, Tokyo, Japan, 1995.

26. TOUSSAINT, S., On the Existence of Equilibria in Economies with Infinitely Many Commodities and without Ordered Preferences, J. Econ. Theory ss (1984), 98-115.

27. TULCEA, C. I., On the Equilibriums of Generalired Games, The Center for Mathematical Studies in Economics and Management Science, Paper No. 696, 1986.

28. TULCEA, C. I., On the Approximation of Upper-Semicontinuous Correspondences and the Equilibriums of Generalised Games, J. Math. Anal. Appl. 136 (1988), 267-289.

29. YANNELIS, N. C. and RUSTICHINI, A., Equilibrium Points of Non-cooperative Random and Bayesian Games, Positive Operators, Riesz Spaces and Economics (C. D. Aliprantis, K. C. Border and W. A. J. Iuxemburg, eds.), Springer-Verlay, 1991, pp. 23-48.

31. YUAN, X. Z., The KKM Principle, Ky Fan Minimax Inequalities and Fixed Point Theorems, Nonlinear World 2-2 (1995).

32. WAGNER, D. H., Survey of Measurable Selection Theorems, SIAM J. Control Optim. 15 (1977), 859-903. 


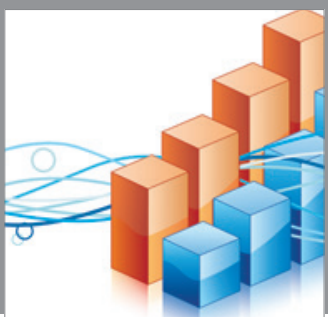

Advances in

Operations Research

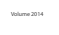

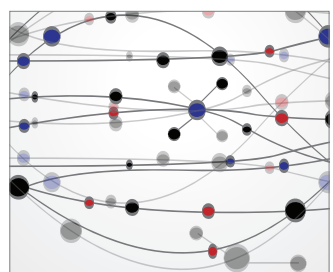

\section{The Scientific} World Journal
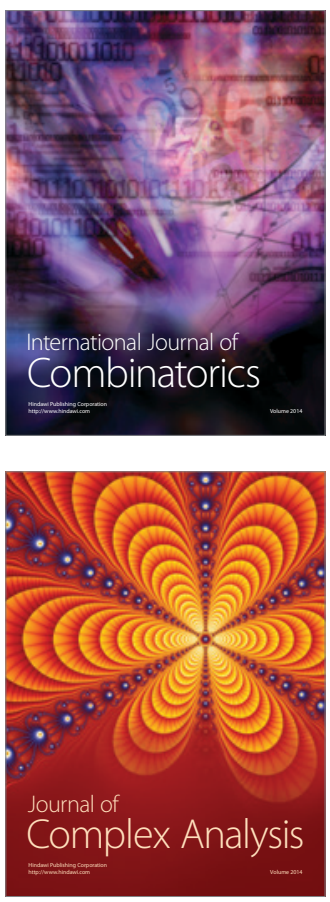

International Journal of

Mathematics and

Mathematical

Sciences
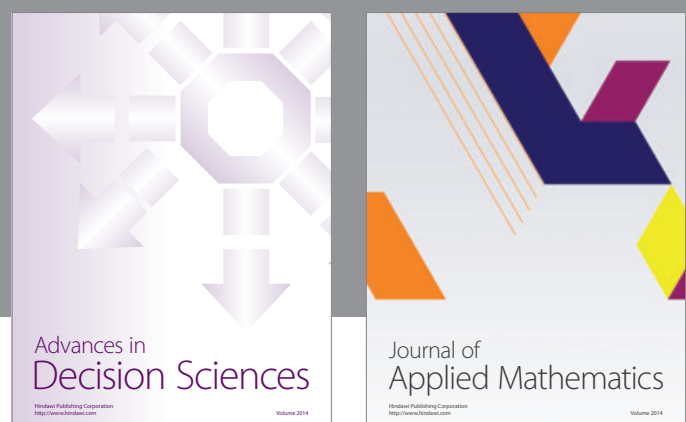

Journal of

Applied Mathematics
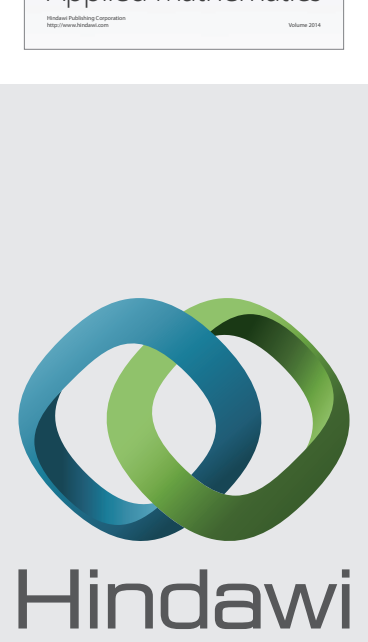

Submit your manuscripts at http://www.hindawi.com
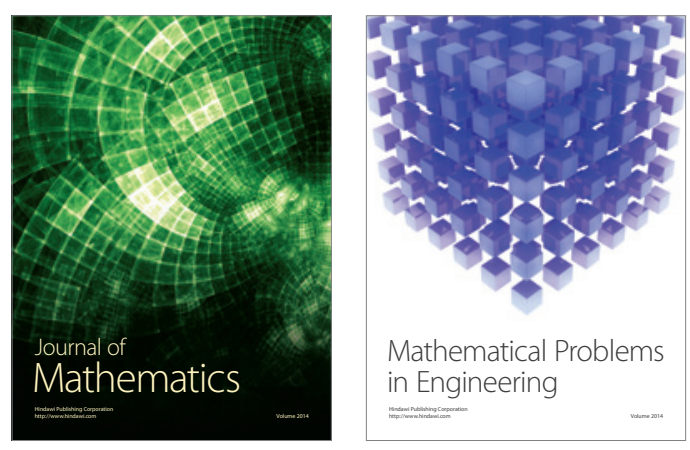

Mathematical Problems in Engineering
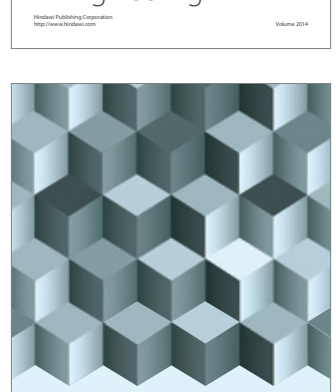

Journal of

Function Spaces
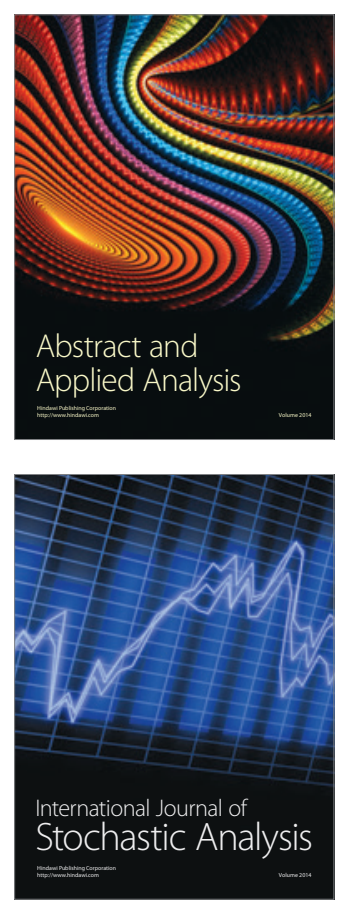

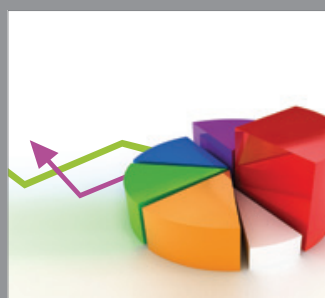

ournal of

Probability and Statistics

Promensencen
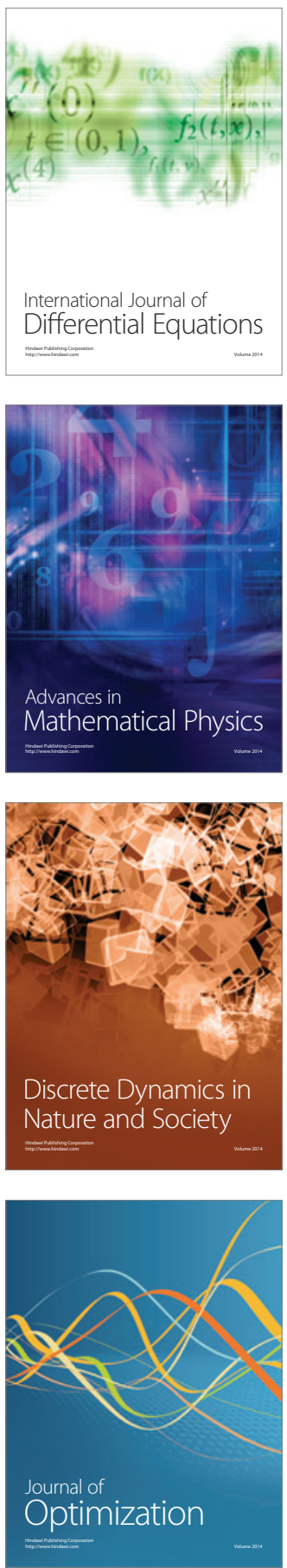